\title{
END SIMPLICIAL VERTICES IN PATH GRAPHS
}

\author{
Marisa GutierRez \\ Conicet \\ Departamento de Matemática, Facultad de Ciencias Exactas \\ Universidad Nacional de La Plata, Argentina \\ e-mail: marisa@mate.unlp.edu.ar
}

AND

Silvia B. Tondato

Departamento de Matemática, Facultad de Ciencias Exactas

Universidad Nacional de La Plata, Argentina

e-mail: tondato@mate.unlp.edu.ar

\begin{abstract}
A graph is a path graph if there is a tree, called $U V$-model, whose vertices are the maximal cliques of the graph and for each vertex $x$ of the graph the set of maximal cliques that contains it induces a path in the tree. A graph is an interval graph if there is a $U V$-model that is a path, called an interval model. Gimbel [3] characterized those vertices in interval graphs for which there is some interval model where the interval corresponding to those vertices is an end interval. In this work, we give a characterization of those simplicial vertices $x$ in path graphs for which there is some $U V$-model where the maximal clique containing $x$ is a leaf in this $U V$-model.
\end{abstract}

Keywords: chordal graphs, clique trees, path graphs.

2010 Mathematics Subject Classification: 05C75.

\section{REFERENCES}

[1] J.R.S. Blair and B.W. Peyton, On finding minimum-diameter clique trees, Nordic J. Comput. 1 (1994) 173-201. 
[2] F. Gavril, The intersection graphs of subtrees in trees are exactly the chordal graphs, J. Combin. Theory Ser. B 16 (1974) 47-56. doi:10.1016/0095-8956(74)90094-X

[3] J. Gimbel, End vertices in interval graphs, Discrete Appl. Math. 21 (1988) 257-259. doi:10.1016/0166-218X(88)90071-6

[4] B. Lévêque, F. Maffray and M. Preissmann, Characterizing path graphs by forbidden induced subgraphs, J. Graph Theory 62 (2009) 369-384. doi:10.1002/jgt.20407

[5] C. Monma and V. Wei, Intersection graphs of paths in a tree, J. Combin. Theory Ser. B 41 (1986) 141-181. doi:10.1016/0095-8956(86)90042-0

[6] Y. Shibata, On the tree representation of chordal graphs, J. Graph Theory 12 (1988) 421-428. doi:10.1002/jgt.3190120313

[7] J.R. Walter, Representations of chordal graphs as subtrees of a tree, J. Graph Theory 2 (1978) 265-267. doi:10.1002/jgt.3190020311

Received 28 November 2013

Revised 17 July 2015

Accepted 17 July 2015 